\title{
La gerencia sostenible como modelo de responsabilidad social
}

\author{
Sustainable management as a social \\ responsibility model
}




\title{
La gerencia sostenible como modelo de responsabilidad social ${ }^{1}$ Sustainable management as a social responsibility model
}

\author{
Javier Francisco Rueda Galvis², Alberto Herrera Guzmán³ ${ }^{3}$ Mónica Andrea Rueda Galvis ${ }^{4}$
}

Artículo recibido en octubre 17 de 2019; artículo aceptado en noviembre 19 de 2019

\begin{abstract}
Este artículo puede compartirse bajo la Licencia Creative Commons Atribución-NoComercial-Compartirlgual 4.0 Internacional y se referencia usando el siguiente formato: Rueda, J. F., Herrera, A. \& Rueda, M. A. (2020). La gerencia sostenible como modelo de responsabilidad social. I+D Revista de Investigaciones, 15 (1), 94-106. DOI: https://doi.org/10.33304/revinv.v15n1-2020010
\end{abstract}

\begin{abstract}
Resumen
La gerencia sostenible es un modelo de gestión capaz de plantear alternativas de solución a muchas de las problemáticas empresariales, bajo un enfoque estructural que entrelaza el crecimiento económico de la organización con el concepto de responsabilidad social. Por esta razón, su implementación se convierte en una estrategia necesaria para contribuir a la sustentabilidad del planeta. En este sentido, el presente documento expone un análisis conceptual y descriptivo de los aspectos más relevantes que involucra la gerencia sostenible como modelo de gestión eficaz, a fin de impulsar desde el ámbito académico la necesidad e importancia de adoptar este tipo de estrategias que mejoran tanto la productividad como la competitividad de la empresa, a la vez que benefician a la sociedad y el medioambiente.
\end{abstract}

Palabras clave: Gerencia sostenible, responsabilidad social, modelo de gestión sostenible.

\begin{abstract}
Sustainable management is a model capable of proposing alternative solutions to many the business problems, under a structural approach that links the economic growth of the organization with the concept of social responsibility. This is the reason why its implementation becomes a necessary strategy to contribute to the sustainability of the planet. In this sense, this document presents a conceptual and descriptive analysis of the most relevant aspects of sustainable management as an effective management model, in order to promote the need and importance of adopting such strategies from the academic field, since they improve both productivity and competitiveness of the company, while benefiting society and the environment.
\end{abstract}

\footnotetext{
${ }^{1}$ Artículo de revisión, de enfoque cualitativo, resultado de un proyecto de investigación terminado, perteneciente al área de ciencias administrativas, subárea responsabilidad social empresarial, desarrollado por los grupos de investigación PORTER, de la Universidad de Investigación y Desarrollo (Bucaramanga, Colombia), GESOE, de la Universidad de La Salle (Bogotá, Colombia), y ORGES 4.0, de la Universidad Cooperativa de Colombia (Bogotá, Colombia). Fecha de inicio: mayo de 2019. Fecha de terminación: octubre de 2019.

${ }^{2}$ Ph. D. en Administración de Empresas, integrante del grupo de investigación PORTER, profesor investigador, Universidad de Investigación y Desarrollo (Bucaramanga, Colombia). Dirección: calle 9 n. ${ }^{\circ}$ 23-55. PBX: 6352525. ORCID ID: https://orcid.org/0000-0002-2795-7844. Correo electrónico institucional: jrueda31@udi.edu.co.

${ }^{3} \mathrm{Ph}$. D. en Administración de Empresas, integrante del grupo de investigación GESOE, profesor investigador, Universidad de la Salle (Bogotá, Colombia). Dirección: carr. ${ }^{a} 2$ n. ${ }^{\circ}$ 10-70. PBX: 3488000. ORCID ID: https://orcid.org/00000-0003-1310-1022. Correo electrónico institucional: aherrera@unisalle.edu.co.

${ }^{4}$ Magíster en Economía, integrante del grupo de investigación ORGES 4.0, profesora investigadora, Universidad Cooperativa de Colombia (Bogotá, Colombia). Dirección: Avenida Caracas n. ${ }^{\circ}$ 45a-55. PBX: 33235652525. ORCID ID: https://orcid.org/0000-0002-4196-9331. Correo electrónico institucional:marueda@campusucc.edu.co.
} 
Keywords: Sustainable management, social responsibility, sustainable management model.

\section{Introducción}

Con base en la problemática actual que vive el planeta en términos ambientales, sumada al efecto de cambio climático y a que la población mundial superó 7500 millones de personas (Census, 2019), resulta oportuno que como sociedad global entendamos que el coexistir de forma sostenible y sustentable es una prioridad que debe solucionar esta generación, en la medida que, de no realizar cambios transcendentales, es muy probable que en menos de 200 años no contemos con recursos naturales suficientes para satisfacer las necesidades de consumo. Igualmente, con base en el aumento poblacional exponencial, el consumismo irresponsable, la desprotección del medioambiente y la falta de visión colectiva clara sobre el futuro de la humanidad, científicos como Stephen Hawking afirman que en menos de 500 años la humanidad no sobrevivirá, por haber convertido al planeta en un escenario apocalíptico y sin recursos (La Gaceta, 2017).

En este sentido, reconocer esta problemática debe ser la primera condición para comprender que los conceptos de desarrollo económico, bienestar social, crecimiento sostenido y calidad de vida están ligados en forma directa con la preservación de los recursos del planeta y la conservación de ecosistemas. Por tanto, es momento de empezar a consolidar un nuevo modelo de pensamiento y gestión empresarial que sea capaz de reconocer la importancia actual y futura que significa para la humanidad la preservación y conservación del medioambiente, como elemento fundamental para alcanzar un desarrollo sostenible que reduzca los problemas sociales y económicos de la población.

En definitiva, esperar acciones efectivas por parte de los gobiernos actuales es una idea poco factible, por lo que son las empresas y la sociedad en general quienes están llamados a liderar procesos de cambio que contribuyan a crear un verdadero entorno sostenible, que sea capaz de entregar a las siguientes generaciones un planeta en iguales o mejores condiciones que el que recibimos. Significa entonces que estrategias asociadas con la gerencia sostenible pueden ser el primer gran paso que se necesita dar para reconstruir el actual panorama que se visualiza para el futuro del planeta, en la medida que es oportuno y necesario lograr hacer compatibles los propósitos de crecimiento económico y desarrollo empresarial, sin que ello genere grandes impactos ambientales en un modelo de convivencia sustentable.

\section{Metodología}

El presente documento corresponde a un artículo de revisión que surge como resultado del análisis de diversas investigaciones preliminares que estudian la problemática ambiental del mundo moderno y posibles soluciones en función del concepto de desarrollo sostenible. Para ello se utilizó la metodología de investigación evaluativa, bajo el esquema de revisión documental, a fin de generar posturas y reflexiones de orden crítico basadas en argumentaciones de carácter bibliográfico que dieran relevancia a la temática expuesta en el ámbito internacional, a fin de generar autoevaluaciones empresariales, como debates académicos, con base en las evidencias aquí descritas (Guirao, 2015).

En términos generales, este artículo presenta un estudio exploratorio de carácter crítico que se soporta en los diversos postulados de orden científico y académicos que analizan el papel de las organizaciones en función de lograr un desarrollo sostenible. Esto, desde un enfoque administrativo que considere la gestión sostenible como un modelo viable que representa soluciones frente a las delicadas condiciones ambientales por las que se encamina el planeta. Con base en este esquema, el presente estudio inició a partir de enunciar una pregunta de investigación sobre la cual se estructurará el desarrollo investigativo para la construcción de un marco conceptual y el análisis de información obtenida: ¿qué estratégicas administrativas deben implementar las organizaciones actualmente para contribuir a lograr un desarrollo sostenible?

\section{Marco conceptual}

\section{Contextualización del desarrollo sostenible}

El término desarrollo sostenible se establece por primera vez en 1987, por la Comisión Mundial para el Medio Ambiente de la Organización de las Naciones Unidas ONU, a través del "Informe Brundtland", con los aportes de 500 investigadores provenientes de 21 países. Con este informe, se analizó el propósito de lograr un equilibrio efectivo entre el desarrollo económico empresarial y la sostenibilidad del planeta, dadas las graves consecuencias que se generarían para el medioambiente y la sociedad, especialmente en los países menos desarrollados (ONU, 2016; FajardoUrbiña, 2018). Desde entonces, el concepto de desarrollo sostenible ha abierto el debate sobre la necesidad de formular propuestas realistas para enfrentar el impacto ambiental que se desprende del consumismo actual, así como nuevas formas de cooperación internacional que estructuren políticas y compromisos por parte de las organizaciones, empresas, gobiernos y sociedad. 
En forma puntual, el desarrollo sostenible se define como "un modelo de crecimiento económico global que satisface las necesidades actuales de la humanidad, sin comprometer la capacidad de las generaciones futuras, para satisfacer sus propias necesidades" (ONU, 1987). Este concepto implica no solo temas de carácter socioeconómicos y empresariales, sino que vincula la supervivencia para la especie humana. Es importante resaltar que el significado de sostenibilidad se debe asociar con el resultado u objetivo que se debe alcanzar a través del desarrollo sostenible, lo que significa que es necesario llevar a cabo acciones que propicien en todos los entornos la responsabilidad social, producción limpia, consumo responsable, tecnologías verdes, cultura ambiental y educación. Estos dos últimos son los elementos más importantes de este modelo (Fremman, 1984; Serna-Mendoza, 2017; Vives, Corral \& Isusi, 2005).

Es importante destacar que el significado de desarrollo sostenible vincula cuatro elementos básicos: sociedad, medioambiente, cultura y economía, lo que evidencia que su enfoque no solo está en el campo de lo ambiental, sino que trasciende hacia un concepto de carácter global que implica la búsqueda del bienestar de la población dentro de escenarios de armonía en cuanto al uso de los recursos naturales (UNESCO, 2014). De estos elementos, definitivamente el factor que más impacto positivo puede generar para alcanzar la sostenibilidad es la cultura, dado que vincula el pensamiento colectivo y se refleja en acciones sociales enmarcadas por normas y códigos de conducta (Cubero-Pérez \& SantamaríaSantigoa, 2005).

Por tanto, el desarrollo sostenible solo será posible si se crean entornos que propicien este tipo de cultura en el hogar, comunidad, gobierno y empresas, de manera tal que nuestro comportamiento se condicione a lograr satisfacer las necesidades mediante actividades que sean coherentes con el desarrollo económico y la sostenibilidad del medioambiente (Rueda, 2017). Lo anterior implica que para crear este fenómeno cultural es necesario que surja un compromiso de cambio y reflexión soportado en una amplia participación ciudadana, a través del cual se integre lo ecológico, económico y social como estructura base de un desarrollo sostenible soportable, viable y equitativo (ver Figura 1), con estrategias que mejoren las condiciones de calidad de vida y de bienestar de las actuales y futuras generaciones.

Significa entonces que analizar la relación existente entre el cambio de estrategia en las organizaciones y las nuevas propuestas basadas en sustentabilidad, son conceptos generadores de creación de valor compartido, en razón a que representan innovación y valor social, tal y como lo plantean Porter y Kramer (2006). Por tanto, una visión compartida y cooperada en la que los agentes involucrados tengan un papel protagónico en el desarrollo económico y social es hoy en día una estrategia de valor compartido, que representa una nueva manera de hacer negocios, en la que el objetivo del lucro empresarial ya no es la única razón de ser de la compañía, sino más bien una consecuencia rentable de crear progreso y beneficios a nivel general para la sociedad.

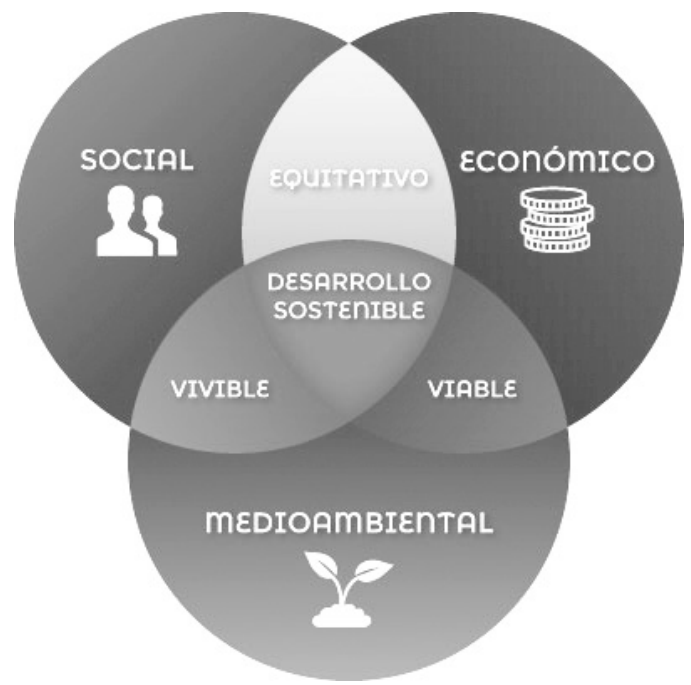

Figura 1. Principios del desarrollo sostenible. Fuente: Tomado de Líneaverde (2018).

En consecuencia, Porter y Kramer (2011) proponen que las organizaciones deben reformular su esquema de crecimiento y desarrollo económico con base en "reinventar su estrategia en dirección a la innovación", a partir de la creación de valor compartido, con lo cual también se impulsa la responsabilidad social de la empresa. Satisfacer las necesidades sociales que define el mercado y contribuir con beneficios a la sociedad es una forma rentable y sostenible de retribuir a la comunidad los múltiples recursos utilizados dentro del esquema de valor compartido, a la vez que se es coherente con los Objetivos de Desarrollo Sostenible (ODS), y se apoya la sostenibilidad del planeta desde un enfoque menos técnico y más humano (ONU, 2018).

En concreto, para lograr el equilibrio correcto entre el uso de los recursos naturales, el impacto en el medioambiente y el desarrollo económico de las naciones, los grupos de interés o stakeholders y organizaciones deben crear espacios de concertación en donde se puedan construir estrategias satisfactorias a cada una de las partes negociadoras, en función del respeto a las normas legales establecidas y los principios culturales de armonía y convivencia entre ser humano y los ecosistemas del planeta. Es por esto que se debe analizar cómo los modelos productivos y de consumo se ajustan a los dos enfoques centrales del desarrollo sostenible, que plantean acciones que mejoren eficazmente la calidad de vida de las personas, al 
tiempo que custodian la integridad de los sistemas que sustentan la vida. Esto, dentro de un marco que permita satisfacer las necesidades de la sociedad y los objetivos económicos empresariales con un aprovechamiento coherente de los recursos naturales (Carpenter, 1991).

Para que el desarrollo sostenible consiga alcanzar el propósito de la sostenibilidad, 189 países integrantes de la ONU, a través del Programa de las Naciones Unidas para el Desarrollo (PNUD, 2016), establecieron 17 metas para el año 2030 que son reconocidas como los Objetivos de
Desarrollo Sostenible (ODS) (ver Figura 2). Lo anterior, con el propósito fundamental de lograr "eliminar la pobreza, protegiendo el planeta en paz y con prosperidad". Esta agenda global 2030 representa uno de los retos más importantes para la historia de la humanidad, ya que más de 1000 millones de personas viven en condición de pobreza extrema (menos de US\$1,25 al día) y cerca de 2800 millones no cuentan con acceso al agua potable, en un planeta que utiliza $90 \%$ del combustible fósil generando gases de efecto invernadero, calentamiento global y destrucción de ecosistemas.

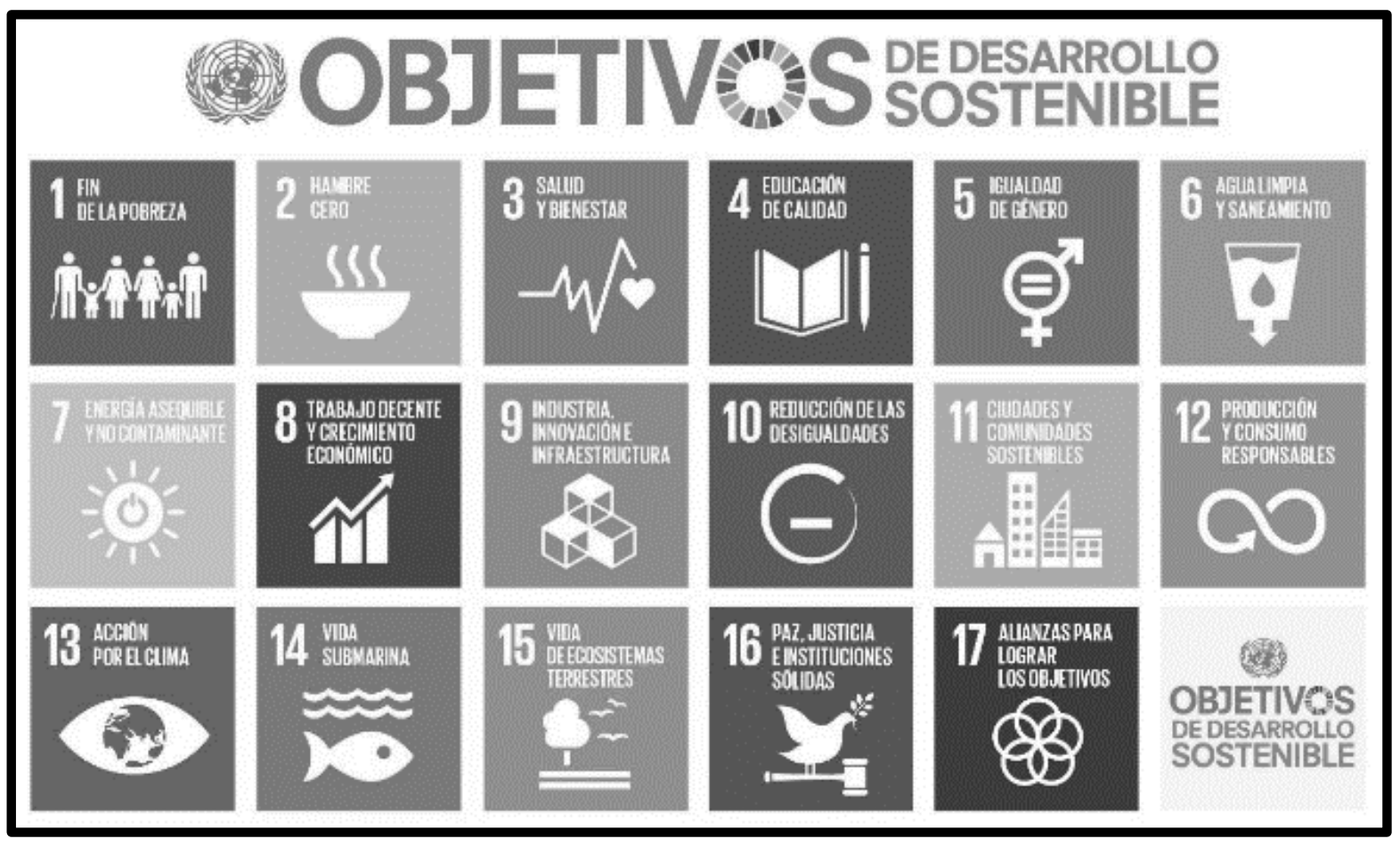

Figura 2. Objetivos de Desarrollo Sostenible. Agenda 2030 Fuente: Acciona (2017).

Uno de los objetivos más complejos de los ODS es logar contar con ciudades y comunidades sostenibles, en la medida que más del $60 \%$ de la población mundial actual vive en centros urbanos, y es allí donde se generan mayores niveles de impacto ambiental puntuales. Este problema crece constantemente; la ONU (2016) proyecta que en 2050 en estos espacios vivirán 6500 millones de personas. Esto implica que comunidades, organizaciones y gobierno adopten modelos de crecimiento basados en consumos responsables que posibiliten la reducción de la huella ecológica que producen las personas y los procesos empresariales, a fin de minimizar los impactos ambientales y factores de cambio climático. Este fenómeno se ha incrementado en aproximadamente $50 \%$ desde el 2015, en comparación con 1990; se ha aumentado en $2{ }^{\circ} \mathrm{C}$ la temperatura del planeta en el último siglo, lo que en forma natural se da cada 5000 años (Gómez \& Gómez, 2013).
Es de destacar que el último OSD implica la creación de estrategias mundiales mediante alianzas, mecanismos de participación y cooperación internacional. Se reconoce que la problemática ambiental ya no es exclusiva de un país, sino que implica la afectación del planeta entero $y$, por ende, de toda la humanidad. Es aquí cuando las empresas y organizaciones deben estructurar nuevos modelos de gestión que involucren estrategias de crecimiento en escenarios sostenibles que promuevan la conservación de los ecosistemas y sus recursos naturales. Esto, dentro de la estructura comercial y productiva que enmarca la globalización de los mercados con sus asimétricas, particularmente en el tema ambiental, dados los sistemas de fabricación a gran escala que involucran procesos contaminantes sin control, así como las escasas regulaciones internacionales y el marcado fomento al consumismo (Sachs, 2001; Goldin \& Reinert, 2005; Kendall \& Kendall, 2005; Méndez-Francisco, 2007; Romero, 2002; Bresser, 2014). 
En el tema ambiental, la globalización genera impactos altamente negativos para la biodiversidad, dada la gran escala de procesos extractivos y productivos que se realizan en cada ecosistema, en respuesta a la creciente demanda que impone el consumismo año a año a nivel del planeta (Jenkins, Pimms, \& Joppa, 2013; OchoaGarcía, 2015; Fernández, 2009; Goldin \& Reinert, 2005); Instituto \& Humboldt, 2012). Autores han señalado que la sobreexplotación de recursos naturales es la mayor fuente de afectación y destrucción de los ecosistemas y su biodiversidad, como consecuencia de los escasos controles y una marcada incultura ambiental, lo que, por ejemplo, hace que el $75 \%$ de los recursos pesqueros del mundo estén actualmente sobreexplotados. Esta situación significará en el 2050 la desaparición de más del $90 \%$ de las especies marinas, y solo la agricultura, en menos de 50 años, será la única fuente de alimentos, tal y como lo afirma la Organización de las Naciones Unidas para la Agricultura y la Alimentación (FAO, 2015).

Soporte de lo anterior es el Informe Científico de IPBES, (2019), con más de 1800 páginas, que ofrece datos irrefutables sobre la condición de deterioro y caos que está causando el ser humano a la naturaleza y sus ecosistemas en todo el mundo, lo que se evidencia especialmente con más de 1 millón de especies animales y vegetales que están actualmente en peligro de extinción. Este contexto pone a la humanidad en un punto de no retorno. Igualmente, el estudio reitera un alarmante agotamiento de los recursos y servicios de los ecosistemas para el sustento humano y para el desarrollo económico: $75 \%$ del ambiente terrestre y $66 \%$ del marino han sido alterados drásticamente. Además, en la actualidad el $33 \%$ de la superficie terrestre y cerca del $75 \%$ del agua dulce se dedican exclusivamente a la producción agrícola o ganadera, con una emisión de gases de efecto invernadero duplicada y aumento del nivel del mar de 16 a 21 centímetros desde 1900 .

Sumado a lo anterior, la incontrolada deforestación de bosques en el ámbito mundial hace que desde 1990 a nuestros días hayan desaparecido más de 130 millones de hectáreas, con una tasa de crecimiento de 15 millones de hectáreas por año sin ningún tipo de control. Los bosques tropicales son los más afectados, con cerca de 450 millones de hectáreas que han sido taladas tan solo en la última década (FAO, 2015). Por estas razones, pensar en conceptos relacionados con sistemas de economía sostenible o economía ambiental (Hartwick, 1977) para que las organizaciones adopten estrategias que permitan el mantenimiento del stock de capital ambiental como modelo de negocios y obligación moral con los ecosistemas que afectan es una idea que debe impulsarse cada vez más (Friedman, 2005).

Como lo expresa Bono (2012), en el último siglo el desarrollo humano dependía del éxito empresarial que impulsaba a su vez la economía de las naciones. Esta situación actualmente resulta insostenible, en términos ecológicos y sociales, lo que afectará las condiciones que mantenían lo que reconocemos como progreso. Por tal razón, plantea que ante esta situación es necesario redimensionar el concepto de prosperidad basada en lo material por nuevas ideas socioeconómicas que incluso puedan analizar la posibilidad de lograr un decrecimiento sostenible, a través del cual se logre el desarrollo humano en condiciones económicas basadas en valores humanos de solidaridad e igualdad. Esta solución contribuye a respetar los límites ecológicos y necesidades de las generaciones en función de los recursos del planeta.

En este sentido, al lograr una economía sostenible las empresas comprenden que asumir el costo de mantener sus recursos naturales es una estrategia generadora de utilidades de mediano y largo plazo que permite crecimiento estable y sostenido, a la vez que se reducen costos y se logra mayor impacto social, en la medida que se contará con recursos en el futuro que ahora son escasos, costosos o poco sustituibles, de forma que el modelo financiero sea rentable y se satisfaga el fenómeno de oferta y demanda. Para ello, es necesario que se realice una valoración del capital ambiental de los recursos naturales renovables y no renovables utilizados para saber su valor de reposición, lo que se asuma como una inversión de compensación real que permitirá la sustentabilidad de los bienes ofrecidos y la conservación del medioambiente (Svartzman, 2015).

En otras palabras, la economía sostenible es un concepto ético y financiero que valora los recursos naturales y sus ecosistemas como un activo que representa un inventario limitado de bienes, para poder hallar el nivel de stock óptimo tanto de uso como de reserva, en términos de niveles máximos y mínimos permisibles, a fin de lograr condiciones de sostenibilidad y abastecimiento permanente (Castiblanco, 2007). El gran aporte de la economía ecológica es que tiende, a través de procesos cuantitativos, a alcanzar un equilibrio entre crecimiento económico y sostenibilidad de los recursos ambientales, con base en tres estrategias:

- Optimización en la explotación de los recursos naturales.

- Optimización de los medios de gestión ambiental.

- Optimización de los instrumentos para alcanzar el desarrollo sostenible.

La valoración de un recurso natural implica realizar el cálculo correcto del costo que representa el bien natural y el costo de su utilización, en el corto, mediano y largo plazo (Costanza et al., 1997; Svartzman, 2015), en función del precio de mercado y del valor de los beneficios que este genera en un ecosistema. Para determinar el valor de un recurso ambiental, existen diversas técnicas, como 
los métodos directos, que se focalizan en estimar rublos y precio de mercado con base en el costo de las alternativas de conservación o recuperación de los recursos naturales, en función del nivel de impacto ambiental (Departamento Técnico Administrativo del Medio Ambiente [DAMA], 1997).

En cuanto a métodos indirectos o también conocidos como de preferencias reveladas, se utilizan como argumentos complementarios bajo los parámetros del costo de reposición, función de producción, valor recreativo y precios hedónicos o de placer. Este tipo de mediciones permite tener una valoración de flujos futuros de capital y del stock de inventario de los activos ambientales, información que es el soporte para calcular la huella de carbono (medición de emisiones de dióxido de carbono CO2) y la huella ecológica. Esta última es el indicador que evalúa el impacto específico que se genera en el entorno, en función de la biocapacidad natural del ecosistema para autoregenerarse, que para el 2005 se estableció en 2,7 hectáreas globales por persona, lo que supera el 0,6 hag determinado como valor promedio que debería tener cada habitante en el planeta (Castiblanco, 2007; Espinoza, 2001).

\section{La gerencia sostenible como modelo de gestión empresarial}

A partir de los enunciados que formula la economía ambiental, las organizaciones pueden implementar nuevos modelos de gestión que resulten ser más eficientes en términos de estrategias, para alcanzar un verdadero desarrollo sostenible, por lo que este enfoque administrativo actualmente se reconoce dentro del concepto de la gerencia sostenible. Involucrar en la empresa la sostenibilidad como un elemento estratégico que se encarna en las políticas, modelo de gestión y cultura corporativa es sin lugar a dudas una de las fuentes más importantes para la generación de beneficios y recursos económicos, dado que envuelve no solo los intereses de la organización, sino también los de sus grupos de interés o stakeholders (Freeman \& Reed, 1983; Glasson, Therivel, \& Chadwick, 2016; Naredo, 2002; Robbins \& Judge, 2009).

Es por esto que la gerencia sostenible se convierte en un modelo de negocios que logra crea valor agregado para la compañía y sus stakeholders, en la medida que se convierte en un factor competitivo que logra un crecimiento sostenible y facilita mejores condiciones para alcanzar el éxito empresarial, en función de una mayor responsabilidad social frente al medioambiente y mejores resultados financieros, gracias a una mejor percepción de la imagen corporativa (Atkinson, Waterhouse, \& Wells, 1997; Empresarial, 2003; Rueda-Galvis \& RuedaGalvis, 2017). Bajo el concepto de gerencia sostenible, las organizaciones desarrollan un modelo de negocios e inversiones innovadoras con lineamientos estratégicos de mediano y largo plazo, que se ajustan a los objetivos corporativos de crecimiento financiero y sostenibilidad bajo una administración eficiente de los recursos y la satisfacción del cliente con demandas futuras de productos o servicios (Carroll, 1991; Schvarstein, 2003).

Como lo expresan Paz-Marcano, Sánchez-Gonzalez y Sánchez-Valbuena (2018), la responsabilidad social se fundamenta en la inclusión de estrategias de gestión que estén enfocadas en alcanzar una mayor competitividad y cohesión social, en la medida que con ello se logra un factor de diferenciación con ventajas competitivas para el mercado y un balance ético organizacional. De igual manera, Pelekais y Aguirre (2008) establecen que la responsabilidad social se soporta en los valores y principios éticos reguladores del maniobrar propio de cada empresa, mediante una acción voluntaria que se expresa en su modelo de gestión con estrategias y compromisos ante sus grupos de interés en actividades que permitan mejorar los niveles de excelencia.

Como lo describen Argandoña (1998) y Arbaiza (2015), el objetivo central de la gerencia sostenible es establecer dentro de su misión corporativa un enfoque global, a través del cual se promueven cambios significativos con beneficio mutuo, tanto para la organización como para su entorno socioambiental, desde un contexto mercantil que se ajusta a las exigencias éticas y morales que impone la responsabilidad social y el compromiso de contribuir a un desarrollo sostenible real. Para implementar un modelo de gerencia sostenible, la empresa debe formular estrategias de alto nivel basadas en valores corporativos centrada en el beneficio particular y colectivo, como una forma diferente y rentable de hacer negocios en sinergia con los intereses de los stakeholders (ver Figura 3).

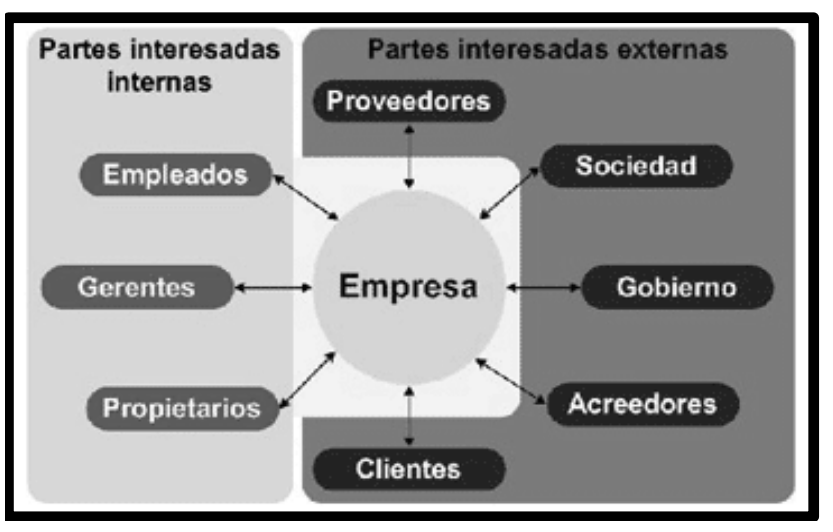

Figura 3. Grupos de interés o stakeholders. Fuente: IFEF Instituto de Fomento (2016).

En este sentido, la razón por la que las empresas fracasan al implementar un modelo de gestión sostenible se basa en que no existe una visión corporativa de largo plazo, al tiempo que no existe una participación de los stakeholders en el proceso de toma de decisiones ni una cultura corporativa que comprenda los conceptos de sostenibilidad 
y sustentabilidad. Por tanto, implementar una gestión sostenible implica la adopción de estrategias basadas en políticas de responsabilidad social que construyan propuestas conjuntas entre empresa y stakeholders, en función de esquemas normativos como la ISO 14001 e ISO 26000 como modelos que contribuyen a un eficiente desarrollo sostenible, con lineamientos estratégicos en función de lo ecológico, económico y social, como elementos que aportan valor competitivo a la organización (Alemán-Santillán, 2005; Vanegas-López, 2011).

Como lo exponen López, López e Ignacio (2005), los modelos de gerencia sostenible son una forma segura de impulsar la competitividad empresarial cuando las empresas visualizan un horizonte de mediano y largo plazo. Lo anterior, en la medida que logran la optimización de sus procesos; mayor sustentabilidad de los recursos; mejor imagen corporativa (Good Will); productos más amigables con el medioambiente; mayor satisfacción de sus grupos de interés; incremento de la fidelización de clientes y la rentabilidad de la compañía en los mercados locales e internacionales. Para Amorós, (2007), Fernández (2005) y Martín-López, González, Díaz, Castro y GarcíaLlorente (2007), la condición de ser empresa sostenible implica demostrar en acciones reales los valores y conductas que definen su responsabilidad social, como factor de éxito y elemento de ventaja competitiva que genera valor agregado y se cuantifica como un activo intangible (Kramer \& Porter, 2002).

Para que la gerencia sostenible logre los resultados esperados, es de vital importancia que el modelo de negocio constituya procesos e indicadores que midan los alcances de las estrategias de sostenibilidad que se plantea la organización a través del tiempo. Este esquema debe desarrollar, según el enfoque de cada empresa, diversos elementos básicos de preciso cumplimiento, a través de los cuales se realizarán los procesos administrativos de planeación, organización, dirección y control, estableciendo el logro de las metas y objetivos formulados desde el modelo de la gerencia sostenible (Boada, Toledo \& Artis, 2003).

\section{Resultados de investigación}

\section{Elementos básicos para la formulación de un modelo de gerencia sostenible}

Para iniciar un modelo de gerencia sostenible, se reconoce como primer eje de trabajo el compromiso de la empresa por instaurar y fomentar una cultura empresarial basada en el desarrollo sostenible que reconozca este concepto como una política institucional que está en concordancia con la misión y visión corporativa, a fin de crear un pensamiento colectivo que impulse la competitividad como un resultado no solo de los procesos productivos y comerciales tradicionales, sino también de la optimización de los recursos naturales y la puesta en marcha de programas de responsabilidad social (Cubero-Pérez \& Santamaría-Santigoa, 2005; Robbins \& Judge, 2009).

Como segunda condición, la gerencia sostenible debe promover la participación de los grupos de interés o stakeholders, en la medida que son estos quienes pueden legitimar e impulsar las estrategias que adopte la empresa en pro de un desarrollo sostenible, dentro de un esquema de compromiso sinérgico entre las partes y coherente con los objetivos de éxito de la organización (Argandoña, 1998; Donaldson \& Preston, 1995; Freeman \& Reed, 1983). Es de vital importancia para el modelo la inclusión de los stakeholders, ya que, de su nivel de empoderamiento dependerá el éxito de las estrategias que se pretenden implementar, por lo que deben ser considerados elementos claves del proceso.

Consecuentemente, la empresa debe impulsar desde su modelo de gestión el enfoque de sostenibilidad, a partir del desarrollo de procesos productivos que den total cumplimiento de las normas ambientales, tanto en el ámbito local como en el internacional. Lo anterior, con el objetivo de que, en primera instancia, este accionar sea coherente con los dos postulados anteriores y se pueda crear valor competitivo en nuevos mercados, con base en el respeto por el medioambiente, los ecosistemas y las comunidades vinculantes (Pinto-Saavedra, 2007; Porter, 1994, 1996).

En conexión con lo anterior, es fundamental que la empresa implemente la adopción de estándares internacionales de gestión ambiental que sean acordes a su propósito de sostenibilidad, para lo cual normas como la ISO 9000, ISO 14000 e ISO 26000, entre otras, son esenciales en este sentido. Estas normas otorgan a la organización pautas para su accionar dentro de esquemas de modelos de gestión, a la vez que incrementan su posicionamiento de marca, imagen y productos en el mercado internacional (ISO, 2012; Mijangos-Ricárdez, 2015; Naredo, 2002).

Una correcta gestión sostenible debe establecer un proceso de auditoría y control mediante la presentación periódica de informes de rendición de cuentas, a través de los cuales sea posible visualizar en forma proactiva si los planes y acciones desarrolladas se ajustan al objetivo de lograr un desarrollo sostenible mediante el uso de indicadores (Pacheco, Castañeda \& Caicedo, 2002; Perevochtchikova, 2013). Este proceso de rendición de cuentas es una herramienta de mejoramiento continuo que permite tanto a la empresa como a sus grupos de interés observar los resultados que genera la gerencia sostenible, en función de los entornos económicos, sociales, productivos, ambientales, administrativos y financieros. Esto puede ser de gran beneficio para la compañía, en términos competitivos, si los resultados resultan positivos o demuestran progresos (Acosta- 
Medina, Plata-Gómez, Puentes-Garzón \& Torres-Barreto, 2019; Marín, 2011; Semarnat, 2012).

El objetivo de estas estrategias de gestión sostenible es la búsqueda de una mejor eficiencia ambiental, que, desde la perspectiva de Blanco (2013), Ortega, Sánchez y Ortega (2010), Gil (2011) y Ortega et al. (2010), se refiere a la capacidad que debe tener la organización de gestionar procesos que demanden la cantidad mínima posible de recursos naturales como afectaciones en términos de impactos ambientales. Desde la óptica de Naredo (2002), esta eficiencia ambiental se puede impulsar a partir de la implementación de las normas ISO 9000 e ISO 14000, como parte de un esquema de gestión empresarial que plantea objetivos y estrategias para su evaluación, tal y como se propone en el modelo de gerencia sostenible que se expone en la Tabla 1.

Tal y como lo expresa Quiroga-Martínez (2001), en los estudios realizados para la CEPAL, para que este tipo de modelos gerenciales prospere, es necesario establecer indicadores que midan su efectividad en términos de sostenibilidad ambiental e impacto. Para lograr medir los niveles de sostenibilidad ambiental, es esencial utilizar indicadores básicos, tales como la huella ecológica, huella de carbono, huella hídrica y huella social, como elementos para el mejoramiento de procesos.

El indicador de la huella ecológica se refiere al tiempo necesario para que un área natural pueda producir los mismos recursos que se consumen por una comunidad u organización. Para el caso de la población mundial, actualmente se necesita un año y medio para poder regenerar lo demandado en un año, valor que demuestra que aún no somos sostenibles. En el caso de la huella de carbono, este es otro índice que se utiliza para medir el nivel de gases de efecto invernadero (GEI) que se produce por la fabricación y comercialización de bienes o servicios, en términos de $\mathrm{CO} 2$, con base en los estándares de referencia de la norma ISO 14067.

Tabla 1

Propuesta de modelo de gerencia sostenible

\begin{tabular}{ll}
\hline \multicolumn{1}{c}{ Objetivo } & \multicolumn{1}{c}{ Estrategias } \\
\hline $\begin{array}{l}\text { 1. Incrementar la eficiencia de uso de los } \\
\text { recursos renovables }\end{array}$ & $\circ$ Minimizar el tamaño del stock del recurso natural \\
& $\circ$ Incrementar la producción del recurso renovable a futuro \\
& $\circ$ naturales \\
\hline 2. Incrementar la eficiencia de uso de los & $\circ$ Búsqueda de sustitutos del recurso natural no renovable \\
recursos no renovables & $\circ$ Implementar estrategias de reciclaje para el recurso no renovable mediante la \\
& recuperación de materias primas \\
\hline 3. Reducir los niveles de impacto & $\circ$ Desarrollar mayor cantidad de productos biodegradables \\
ambiental y contaminación & $\circ$ Incrementar los volúmenes de reciclaje de residuos propios \\
& $\circ$ Mejorar el sistema de tratamiento de los residuos finales \\
& $\circ$ Mejorar la capacidad de asimilación de entorno afectado \\
& $\circ$ Diseñar nuevas formas de manejo de desperdicios seguros \\
\hline 4. Optimizar costo de manera eficiente a & $\circ$ Reducir los costos generados por el manejo de residuos \\
partir de administrar los recursos & $\circ$ Reducir el consumo de energía empleada en los procesos \\
consumidos o los residuos descargados & $\circ$ Utilizar tecnologías verdes o menos contaminantes \\
& $\circ$ Disminución de recursos usados en producto, empaquetado y distribución \\
& $\circ$ Implementar estrategias de 3R, reducir, reutilizar y reciclar \\
\hline R. Rediseño de los productos, bienesy & $\circ$ Rediseño de materiales, formas y tamaños del producto \\
servicios & $\circ$ Incrementos en la durabilidad del producto \\
& $\circ$ Aceleración de la biodegradación del producto \\
& $\circ$ Reducción del consumo de energía del producto \\
& $\circ$ Diseñar productos totalmente biodegradables \\
& $\circ$ Mejorar la vida útil y calidad del producto \\
\hline 6. Estructurar cambios en el & $\circ$ Creación de culturas de compra enfocadas en el consumo de productos con menor \\
comportamiento de la demanda & contenido material \\
& $\circ$ Incentivar el consumo responsable mediante la demanda de productos más \\
& $\circ$ amigables con el medioambiente \\
& $\circ$ y comentar e incentivar los procesos de reciclaje por parte del proveedor, distribuidor \\
\hline
\end{tabular}

Fuente: Elaboración propia con base en Naredo (2002), Boada y Toledo (2003), Conesa, (1996), Canter (2007), Amorós, (2007), Zúñiga-Palma (2009), ISO (2012), Semarnat (2012) y Mijangos-Ricárdez (2015).

Igualmente, la huella hídrica permite medir la cantidad de agua dulce que demanda una empresa en forma directa e indirecta en sus procesos, así como los niveles de contaminación y recuperación de esta. De esta forma, es un índice vital para la medición de impactos ambientales, y está regulada por la ISO 14046 (ISO 26000, 2010). A manera de ejemplo, la huella hídrica establece que para producir una taza de café se necesita cerca 
de 140 litros de agua limpia; de la misma forma, para 1 tomate $=13$ litros; 1 papa $=25$ litros; 1 vaso de cerveza $=75$ litros; 1 copa de vino $=120$ litros; 1 vaso de leche $=$ 200 litros; 1 hamburguesa $=2400$ litros; 1 kilo de carne de res $=15.000$ litros; $y$ el acero de un auto $=166.000$ litros (Natura Medio Ambiental, 2016).

En paralelo, la huella social es otra herramienta de medición que permite cuantificar el nivel de impacto de la empresa, a partir de variables de responsabilidad social, tales como el número de empleos generados, consumo de recursos, reciclaje de materias primas, reducción de impactos socioambientales y respeto por los derechos fundamentales, entre otros (Quiroga-Martínez, 2001).

En términos bursátiles y financieros, también existen indicadores de sostenibilidad que permiten cuantificar el nivel de desarrollo que alcanza una empresa, de los cuales se destacan principalmente tres: Dow Jones Sustainability Indexes y Domini 400 Social Index, que son usados en Estados Unidos, y el FTSE4 Good, aplicable en Europa. Son una variable de medición para los inversionistas y logran una valoración de la responsabilidad social que alcanzan las empresas (Barcellos, 2011).

En definitiva, lograr estructurar una gerencia sostenible en la organización representa crear escenarios productivos y competitivos en el ámbito internacional, de modo que la empresa logre obtener beneficios significativos de todo sentido, especialmente en lo que se refiere a alcanzar los objetivos estratégicos de orden comercial y financiero, así como una mayor atracción de clientes, inversionistas, eficiencia administrativa, beneficios tributarios, posicionamiento de marca, fidelización de consumidores y una imagen de compañía socialmente responsable. Así, se garantiza la continuidad del negocio y la sustentabilidad de sus recursos al integrar stakeholders y medioambiente.

\section{Conclusiones}

Toda empresa debe reconocerse como una organización capaz de asumir su rol responsable frente a las consecuencias ambientales que generan sus procesos productivos, por lo que es fundamental que su pensamiento administrativo esté impulsado actualmente por una filosofía de responsabilidad social enmarcada en el concepto del desarrollo sostenible. Lo anterior, a fin de construir planes estratégicos de crecimiento económico y éxito empresarial viables que no impliquen el deterioro de las condiciones ambientales de los diversos ecosistemas, ni el consumo sin control de los recursos naturales que ofrece el planeta.

Es necesario impulsar desde la academia la formación de profesionales capaces de cambiar en el ámbito empresarial el paradigma de que el crecimiento económico y la preservación del medioambiente son alternativas opuestas. Está ampliamente demostrado y argumentado que adoptar un modelo de gerencia sostenible es una estrategia generadora de beneficios para los inversionistas y para los objetivos que persiguen los stakeholders. Es por esto que temas como el desarrollo sostenible deben ser un eje temático transversal en todos los niveles de educación y formación, para que se convierta en una cultura ciudadana y se constituya en una forma de pensamiento y de comportamiento social.

Para desarrollar adecuadamente un modelo de gerencia sostenible, es necesario combinar estrategias de cultura corporativa, responsabilidad social, implementación de normas ISO (14000 y 26000) y compromiso organizacional. Esto, a fin de crear un entorno de éxito empresarial con altos estándares de productividad y competitividad, acordes a los cambios que imponen el fenómeno de la globalización de los mercados y los efectos sobre el medioambiente.

Impulsar la gerencia sostenible como modelo administrativo es una forma de generar cambios definitivos en nuestro accionar como sociedad global, preservando nuestra biodiversidad y recursos naturales, en un esquema que permite el crecimiento económico, la satisfacción de necesidades y el mejoramiento de la calidad de vida.

Esta década es el momento oportuno que nos ofrece el planeta para generar estrategias de cambio, porque, de no hacerlo, estaremos condenando a las siguientes generaciones a vivir en un mundo desamparado y con un futuro incierto. De no hacer nada, en aproximadamente 50 años los daños causados en términos ambientales serán en su gran mayoría irreversibles, especialmente en recursos tan valiosos para la supervivencia humana y de las demás especies, como el agua, aire, suelos, capa de ozono y todos los demás ecosistemas que conforman nuestra cada vez más escasa biosfera.

En definitiva, es un cambio de comportamiento individual y colectivo lo que implica poder garantizar un futuro adecuado a esta a y las generaciones venideras. Es razón suficiente para trabajar en pro de mejorar los niveles de eficiencia ambiental a partir de nuestros actos, comprendiendo que somos parte de un único ecosistema en el que los recursos son cada vez más escasos.

Por estas razones, el deber ser de las empresas y su gestión social bajo el concepto de organizaciones competitivas debe ir más allá de simples mediciones a través de indicadores de productividad, rentabilidad o crecimiento económico. Esto, dada su obligación ética y moral de alinear su quehacer dentro de un contexto de responsabilidad social y ambiental que se incluya como ejes estratégicos en los planes de desarrollo a corto, mediano y largo plazo. 
En Colombia, casos empresariales como los de Ecopetrol, que desde 2018 integra a su Global Reporting Initiative (GRI) informes de sostenibilidad; el Grupo Nutresa, que incorpora prácticas con sus stakeholders en temas de medición de impactos ambientales; y Alpina y Davivienda, que generan valor mediante estrategias económicas, ambientales y sociales en pro de la innovación y preservación de los recursos naturales, son una buena muestra de que sí es posible implementar elementos gerenciales sostenibles para impulsar el crecimiento organizacional, con eficiencia, calidad y desarrollo social.

\section{Referencias}

Acciona. (2017). ¿Qué son los objetivos de desarrollo sostenible? Recuperado de https://www.acciona. com/es/desarrollo-sostenible/

Acosta-Medina, J. K., Plata-Gómez, K. R., Puentes-Garzón, D. E., \& Torres-Barreto, M. L. (2019). Influencia de los recursos y capacidades en los resultados financieros y en la competitividad empresarial: una revisión de la literatura. I+ D Revista de Investigaciones, 13(1), 147157. https://doi.org/10.33304/revinv.v13n1-2019013

Alemán-Santillán, T. (2005). Desarrollo sustentable: Teoría y Práctica. Revista Ecosur., 24(1).

Amorós, E. (2007). Comportamiento Organizacional En Busca del Desarrollo de Ve.tajas Competitivas. Universidad Católica de Santo Toribio de Mogrovejo. Escuela de Economía. Recuperado de https://www.academia. edu/10362382/Comportamiento_Organizacional_En_ Busca_del_Desarrollo_de_Ventajas_Competitivas

Arbaiza, L. (2015). La gerencia estratégica con desarrollo sostenible. Lima. Recuperado de https://www.esan. edu.pe/conexion/actualidad/2015/11/06/gerenciaestrategica-con-desarrollo-sostenible/

Argandoña, A. (1998). La Teoría de los Stakeholders y el Bien Común. Barcelona-España. Recuperado de https:// media.iese.edu/research/pdfs/DI-0355.pdf

Atkinson, A. A., Waterhouse, J. H., \& Wells, R. B. (1997). A Stakeholder Approach to Strategic Performance Measuremen. Sloan Management Review; Cambridge, $38(3), 25-37$.

Barcellos, L. (2011). Modelos de gestión aplicados a la sostenibilidad empresarial. Universidad de Barcelona. Recuperado de http://hdl.handle.net/2445/35386

Blanco, J. A. (2013). Aplicaciones de modelos ecológicos a la gestión de recursos naturales. Omniascience.

Boada, M., Toledo, V. M., \& Artis, M. (2003). El planeta, nuestro cuerpo: la ecología, el ambientalismo y la crisis de la modernidad. Fondo de Cultura Económica., (Vol. 194).

Bono, E. (2012). El Decrecimiento Sostenible, Crisis Ecológico-Económica, Desigualdad Y Economía Social. CIRIEC-España, Revista de Economía Pública, Social y Cooperativa, 76, 180-196.

Bresser, L. (2014). Cambio Estructural para la Igualdad. Una Visión Integrada del Desarrollo. Santiago, Chile:
Ediciones CEPAL. Recuperado de https://repositorio. cepal.org/bitstream/handle/11362/36700/1/LCG2604s_ es.pdf

Canter, L. (2007). Manual de Evaluación de Impacto Ambiental: técnicas para la elaboración de los estudios de impacto. México DF, México: McGraw Hill.

Carpenter, S. (1991). Inventing Sustainable Technologies. Ed: J. Pitt y E. Lugo, The Technology of Discovery and the Discovery of Technology. In Proceedings of the Sixth International Conference of the Society for Philosophy and Technology, Society for Philosophy and Technology. (pp. 481-482).

Carroll, A. B. (1991). The Pyramid of Corporate Social Responsibility: Toward the Moral Management of Organizational Stakeholder. Business Horizons, 34(4), 39-48. https://doi.org/10.1016/0007-6813(91)90005-G

Castiblanco, C. (2007). La Economía Ecológica: Una disciplina en busca de autor. Gestión y Ambiente, 10(3), 7-22.

Census (2019). Population Clock. United State Census Bureau Economic Indicators. Recuperado de https:// www.census.gov/popclock/

Conesa, V. (1996). Los Instrumentos de Gestión Ambiental en la Empresa. (M. P. L. S.A., Ed.). España.

Costanza, R., D'Arge, R., Groot, R. de, Farber, S., Grasso, M., Hannon, B., \& Raskin, R. G. (1997). The value of the world's ecosystem services and natural capital. Nature, 387, 253-260. https://doi.org/doi.org/10.1038/387253a0

Cubero-Pérez, M., \& Santamaría-Santigoa, A. (2005). Psicología cultural: una aproximación Conceptual e histórica al encuentro entre Mente y cultura. Avances En Psicología Latinoamericana, 23, 15-31.

Departamento Técnico Administrativo del Medio Ambiente (DAMA). (1997). Valoración del Impacto Ambiental Gran Industria Manufacturera del Distrito Capital. Bogotá, Colombia. (D. . Santa Fe de Bogotá, Ed.). Bogotá-Colombia. Recuperado de http:// documentacion.ideam.gov.co/cgi-bin/koha/opacdetail.pl?biblionumber $=17189$

Donaldson, T., \& Preston, L. E. (1995). The Stakeholder Theory of the Corporation: Concepts, Evidence, and Implications. Academy of Management Review, 25(1), 65-91. https://doi.org/10.2307/258887

Empresarial, A. (2003). El ABC De la Responsabilidad Social Empresarial en Chile y en el Mundo. Santiago-Chile.

Espinoza, G. (2001). Fundamentos de evaluación de impacto ambiental. Santiago-Chile: Centro de Estudios para el Desarrollo-CED.

Fajardo-Urbiña, L. A. (2018). Desarrollo Sostenible. In II Congreso Internacional de Tecnología, Ciencia y Educación para el Desarrollo Sostenible - CITED. México: Centro Universitario CIFE-CORCIEM.

FAO. (2015). La Evaluación de los Recursos Mundiales. Recuperado de Retrieved from Organización de las Naciones Unidas para la Alimentación y la Agrícultura website: http://www.fao.org/search/es/?cx=018170 620143701104933\%3Aqq82jsfba7w\&q=La+Evalua- 
ción+de+los+Recursos+Mundiales.+2015\&cof=FORID\%3A9\&siteurl=www.fao.org\%2Fforest-resources-assessment\%2Fes\%2F\&ref=www.google.com\%2F\&ss=0j0j1

Fernández, A. (2009). Principales amenazas de la biodiversidad.Recuperado de https://www.consumer. es/medio-ambiente/principales-amenazas-de-labiodiversidad.html

Fernández, R. (2005). Administración de la Responsabilidad Social Corporativa. (Thompson, Ed.). Madrid-España.

Freeman, E., \& Reed, D. L. (1983). Stockholders and Stakeholders: A New Perspective on Corporate Governance. California Management Review, 25(3), 88-106. https://doi.org/10.2307/41165018

Fremman, E. (1984). No TitleStrategic Management: A Stakeholders Approach. (C. university Press, Ed.). Boston.

Friedman, T. L. (2005). The World Is Flat: A Brief History of the Twenty-first Century. (S. and G. Farrar, Ed.). New York-EU.

Gil, J. (2011). Criterios de Eficiencia Ambiental en la Gestión de Residuos. In Conferencia en la cumbre sobre la gestión eficiente de residuos y sus efectos sociales, económicos y medio ambientales. Madrid.

Glasson, J., Therivel, R., \& Chadwick, A. (2016). Introduction to environmental impact assessment: Theory and practice. (3 ed). London-New York: Routledge.

Goldin, I., \& Reinert, K. A. (2005). Globalización y Pobreza. (B. Mundial., Ed.). Washington-USA.

Gómez, D., \& Gómez, M. T. (2013). Evaluación de Impacto Ambiental. (Mundi-Prensa, Ed.). España.

Guirao, J. A. (2015). Utilidad y tipos de revisión de literatura. ENE Revista de Enfermería, 9(2). https://doi. org/10.4321/S1988-348X2015000200002

Hartwick, J. M. (1977). Intergenerational Equity and the Investing of Rents from Exhaustible Resources. The American Economic Review, 67(5), 972-974.

IFEF Instituto de Fomento, E. y F. (2016). Grupos de Interés RS - [Ilustración]. Recuperado de https://www.ifef.es/ portalempleo/rsocial/grupos_interes_rse.php

Instituto, \& Humboldt. (2012). Informe sobre el estado de los recursos naturales renovables y del ambiente Componente de biodiversidad, 2010-2011. BogotáColombia.

IPBES. (2019). Informe de Evaluación Global. Recuperado de from https://www.wwf.org.co/?uNewsID=346653

ISO, 26000. (2012). Norma ISO 26000, Visión General del Proyecto. Ginebra-Suiza.

Jenkins, C., Pimms, S., \& Joppa, L. (2013). Patrones globales de diversidad y conservación de vertebrados terrestres. St. Louis, USA: Jardín Botánico de Missouri.

Kendall, K. E., \& Kendall, J. E. (2005). Análisis y diseño de sistemas. (Sexta edic). México: PEARSON EDUCACIÓN,.

Kramer, M. R., \& Porter, M. E. (2002). La ventaja competitiva de la filantropía corporativa. Harvard Business Review, 80(12), 49-62. https://doi.org/https://dialnet.unirioja. es/servlet/articulo?codigo $=1431332$

La Gaceta. (2017). Stephen Hawking le pone fecha al fin del Mundo. Recuperado de http://bit.ly/2VXbYAm
Líneaverde. (2018). El Desarrollo Sostenible - [Ilustración]. ¿Qué es el Desarrollo sostenible? Recuperado de http://www.lineaverdehuelva.com/lv/consejosambientales/conciencia-ambientual/Que-es-eldesarrollo-sostenible.asp

López, C. D., López, E. S., \& Ignacio, A. (2005). Desarrollo sustentable o sostenible: una definición conceptual. Horizonte Sanitario, 4(2).

Marín, M. I. R. (2011). Modelo de sistema de gestión ambiental. Revista Gestión y Ambiente, 14(1), 151-162.

Martín-López, B., González, J. A., Díaz, S., Castro, I., \& García-Llorente, M. (2007). Biodiversidad y bienestar humano: el papel de la diversidad funcional. Ecosistemas, Revista Científica de Ecología y Medio Ambiente, 16(3).

Méndez-Francisco, L. (2007). Globalización y Medio Ambiente. Revista Inafocam de La República Dominicana., 1(1), 23-41.

Mijangos-Ricárdez, O. F. (2015). Sistemas de Gestión Ambiental bajo normas ISO 14000. In Conferencia en el marco de la carrera de Ciencias Empresariales, At Universidad del Papaloapan campus Tuxtepec. México: Universidad de la Sierra Juárez.

Naredo, J. M. (2002). Economía y Sostenibilidad. Revista Latinoamericana Polis. La Economía Ecológica En Perspectiva, Estudios Sobre Política Ambiental En España, 2. https://doi.org/10.32735/S07186568/2002-N2-155

Natura Medio Ambiental. (2016). Inicio agua ¿Cuántos litros de agua se necesitan para producir? ¿Cuántos litros de agua se necesitan para producir? Universidad Católica de Colombia. Recuperado de https://www.natura-medioambiental.com/cuantoslitros-de-agua-se-necesitan-para-producir/

Ochoa-García, C. (2015). Consecuencias de la globalización en el manejo del medio ambiente.

ONU. (1987). Nuestro futuro común. Madrid: Alianza.

ONU. (2016). Informe Brundtland. Recuperado de http:// bit.ly/2FxqLYe

ONU. (2018). Objetivos de Desarrollo Sostenible. Recuperado de ttps://www.un.org/sustainabledevelopment/es/

Ortega, J., Sánchez, V., \& Ortega, R. (2010). Violencia sexual y cortejo juvenil. In Agresividad injustificada, bullying y violencia escolar, 211-232.

Pacheco, J. C., Castañeda, W., \& Caicedo, C. H. (2002). Indicadores integrales de gestión. Bogotá, Colombia: McGraw Hill.

Paz-Marcano, A. I., Sánchez-González, J. A., \& SánchezValbuena, I. J. (2018). Responsabilidad social factor integrador en Universidades Públicas. I+ D REVISTA DE INVESTIGACIONES, 12(2), 39-50. https://doi. org/10.33304/revinv.v12n2-2018004

Pelekais, C. de, \& Aguirre, R. (2008). Hacia una cultura de responsabilidad social. (P. Educación, Ed.).

Perevochtchikova, M. (2013). La evaluación del impacto ambiental y la importancia de los indicadores ambientales. Gestión y Política Pública, 22(2), 283-312. 
Pinto-Saavedra, J. A. (2007). Los Objetivos del Nuevo Milenio y la Responsabilidad Social de las Pyme: Una Metodología de Evaluación. (F. K. Adenauer, Ed.). Bogotá-Colombia.

Porter, M. E. (1994). Ventaja Competitiva, Creación y Sostenimiento de un Desempeño Superior. (México, Ed.). México: Cecsa.

Porter, M. E. (1996). ¿What is Strategy? Harvard Business Review, 74(6), 61-78.

Porter, M., \& Kramer, M. (2011). Valor compartido. Cómo reinventar el capitalismo y crear una oleada de innovación y crecimiento. Harvard Business Review, 89(1), 31-49.

Porter, M. E., \& Kramer, M. R., (2006). Estrategia y sociedad. El vínculo entre ventaja competitiva y responsabilidad social corporativa. Harvard Business Review, 84(12), 42-56.

Programa de las Naciones Unidas para el Desarrollo PNUD. (2016). Objetivos de Desarrollo Sostenible. Recuperado de http://bit.ly/2ZNDQV8

Quiroga-Martínez, R. (2001). Indicadores de sostenibilidad ambiental y de desarrollo sostenible: estado del arte y perspectivas. (CEPAL, Ed.). Santiago-Chile.

Robbins, S. P., \& Judge, T. A. (2009). Comportamiento organizacional. (PEARSON, Ed.) (13th ed.). México: Administración y Economía.

Romero, A. (2002). Globalización y pobreza. (Uninariño, Ed.). Pasto-Colombia.

Rueda-Galvis, J., \& Rueda-Galvis, M. (2017). Modelo econométrico de gestión exitosa para la empresa familiar colombiana. Revista Finanzas y Política Económica., 9(2), 319-344. https://doi.org/10.14718/ revfinanzpolitecon.2017.9.2.6.

Rueda, J. F. (2017). La Responsabilidad Social Empresarial como estrategia que contribuye al éxito corporativo. Responsabilidad Social. Instituto Tecnológico Superior Cordillera.

Sachs, J. (2001). Ventajas y desventajas de la Globalización. Recuperado de https://cepereloyvaquero.files.wordpress. com/2011/10/tarea-documento-globalizacic3b3n.pdf

Schvarstein, L. (2003). La Inteligencia Social de las Organizaciones: Desarrollando las Competencias para el ejercicio Efectivo de la Responsabilidad Social. Argentina: Editorial Paidós.

Semarnat. (2012). Informe de la Situación del Medio Ambiente en México. Compendio de Estadísticas Ambientales. Indicadores Clave y de Desempeño Ambiental. México D.C. Recuperado de https:// apps1.semarnat.gob.mx:8443/dgeia/informe_12/ pdf/Cap0_docs_previos.pdf

Serna-Mendoza, C. A. (2017). Visiones del desarrollo sostenible. (E. de la U, Ed.).

Svartzman, R. (2015). ¿Qué estudia la economía ambiental y cuál es su diferencia con la economía ecológica? In Primer foro interactivo sobre cambio climático en América Latina. Recuperado de http://bit.ly/2YPBpjK

UNESCO. (2014). Hoja de ruta para la ejecución del programa de acción mundial de educación para el desarollo sostenible. Paris-Francia. Recuperado de http://bit.ly/2Ktyyba

Vanegas-López, D. C. (2011). La responsabilidad social en la alta gerencia una decisión de desarrollo sostenible. Tesis de grado, Repositorio, Bogotá D.C.

Vives, A., Corral, A. \& Isusi, I. (2005). Responsabilidad Social de las Pymes Latinoaméricanas. Recuperado de http:// www.cumpetere.com/wp-content/uploads/2018/02/ PyMEs.pdf

Zuñiga-Palma, H. (2009). elaboremos un estudio de impacto ambiental. Bogotá D.C: Alcaldía de Bogotá. 